8, 32, and 52 compared with $\mathrm{PBO}$. Mean changes from baseline $\mathrm{IBDQ}$ scores were consistently greater for $\mathrm{ADA}$ - vs $\mathrm{PBO}$-treated pts (Abstract PWE-253 table 1). In anti-TNF-naïve pts, improvements from baseline for IBDQ were $34 \pm 38$ and $22 \pm 37$ at Wk $8(\mathrm{p}=0.002)$, $33 \pm 43$ and $24 \pm 43$ at Wk $32(p=0.03)$, and $33 \pm 44$ and $23 \pm 42$ at Wk $52(\mathrm{p}=0.02)$ for $\mathrm{ADA}$ and $\mathrm{PBO}$, respectively. IBDQ responder rates were $68 \%$ and $52 \%$ at Wk $8(p=0.004), 42 \%$ and $27 \%$ at Wk 32 $(p=0.006), 32 \%$ and $21 \%$ at Wk $52(p=0.040)$ for $A D A$ and $P B O$, respectively, among anti-TNF-naïve pts.

Abstract PWE-253 Table 1 Improvement in IBDO

\begin{tabular}{lll}
\hline & PBO, $(\mathbf{N}=\mathbf{2 4 6})$ & ADA, $(\mathbf{N}=\mathbf{2 4 8})$ \\
\hline $\begin{array}{l}\text { IBD0 at baseline (mean } \pm \text { SD) } \\
\text { IBD0 (mean } \pm \text { SD) }\end{array}$ & $123 \pm 33$ & $128 \pm 29$ \\
$\quad$ Week 8 & $20 \pm 36$ & $29 \pm 36^{*}$ \\
Week 32 & $20 \pm 41$ & $28 \pm 41^{*}$ \\
Week 52 & $19 \pm 41$ & $27 \pm 42^{*}$ \\
IBD0 response, n (\%) & & \\
Week 8 & $112(45.5)$ & $144(58.1) \dagger$ \\
Week 32 & $54(22.0)$ & $86(34.7) \dagger$ \\
Week 52 & $40(16.3)$ & $65(26.2) \dagger$ \\
Weeks 8, 32, and 52 & $30(12.2)$ & $58(23.4) \dagger$
\end{tabular}

${ }^{*} p<0.05$. $p$ Values from ANCOVA with treatment and prior anti-TNF status as factors and baseline value as covariate.

$\dagger p<0.05$. $p$ Values from Cochran-Mantel-Haenszel test stratified for prior anti-TNF use.

Conclusion For pts with moderate to severe UC who failed conventional therapy, $\mathrm{ADA}$ was more effective than $\mathrm{PBO}$ for inducing and maintaining improvements in HRQOL, as measured by IBDQ through 52 wks.

Competing interests W Sandborn Grant/Research Support from: Abbott, Consultant for: Abbott, G Van Assche Grant/Research Support from: Abbott, R Thakkar Shareholder with: Abbott, Employee of: Abbott, A Lazar Shareholder with: Abbott, Employee of: Abbott, M Kron Shareholder with: Abbott, Employee of: Abbott, M Yang Shareholder with: Abbott, Employee of: Abbott, S Patel Shareholder with: Abbott, Employee of: Abbott, J Chao Shareholder with: Abbott, Employee of: Abbott, P Mulani Shareholder with: Abbott, Employee of: Abbott.

\section{PWE-254 IMPACT OF INDUCTION DOSING ON MAINTENANCE OUTCOME WITH ADALIMUMAB IN CROHN'S DISEASE}

\section{doi:10.1136/gutjnl-2012-302514d.254}

${ }^{1} \mathrm{~J}$-F Colombel, ${ }^{2} \mathrm{P}$ J Rutgeerts, ${ }^{3} \mathrm{~W}$ J Sandborn, ${ }^{4} \mathrm{~W}$ Reinisch, ${ }^{5} \mathrm{E}$ V Loftus Jr, ${ }^{6} \mathrm{~J}$ Tang, ${ }^{7} \mathrm{P} F$ Pollack, ${ }^{7} \mathrm{M}$ Yang, ${ }^{8} \mathrm{~S}$ P Patel, ${ }^{*}{ }^{7} \mathrm{~B}$ Huang, ${ }^{7} \mathrm{~J}$ Chao, ${ }^{7} \mathrm{P} \mathrm{M}$ Mulani. ${ }^{1}$ Centre Hospitalier Universitaire de Lille, Lille, France; ${ }^{2}$ University Hospital of Gasthuisberg, Leuven, Belgium; ${ }^{3}$ University of California, San Diego, La Jolla, California, USA; ${ }^{4}$ Medical University of Vienna, Vienna, Austria; ${ }^{5}$ Mayo Clinic, Rochester, Minnesota, USA; ${ }^{6}$ Analysis Group Inc, Boston, Massachusetts, USA; ' Abbott Laboratories, Abbott Park, Illinois, USA; ${ }^{8}$ Abbott Laboratories Ltd, Maidenhead, Berkshire, UK

Introduction Two induction regimens of adalimumab are used in Crohn's disease (CD): $160 / 80 \mathrm{mg}$ or $80 / 40 \mathrm{mg}$ at Weeks $0 / 2$. We compared long-term efficacy for patients who received $160 / 80 \mathrm{mg}$ vs $80 / 40 \mathrm{mg}$ as induction therapy followed by eow maintenance therapy.

Methods Data were from two randomised, double-blinded, placebocontrolled efficacy and safety trials in moderate to severe CD. EXTEND, a 52-week study in patients with mucosal ulceration, used the 160-/80-mg induction regimen. CHARM, a 56-week study for maintenance of clinical remission, used the 80-/40-mg induction regimen. All patients who started with induction dose and were randomised to eow plus dropouts prior to Week-4 randomisation were included. Missing Crohn's Disease Activity Index (CDAI) scores were imputed with both non-responder imputation (NRI) and last observation carried forward (LOCF). Remission (CDAI
$<150)$ and hospitalisation were compared between induction regimens. To incorporate the correlation between visits for a patient, a logistic regression with the patient-level random intercept using all the time points after Week 4 was constructed to compare likelihood of remission, controlling for baseline CDAI, fistula, prior use of an anti-tumour necrosis factor therapy, concomitant medications, CD duration, and other factors.

Results 70 patients in the $160-/ 80$-mg group were compared with 336 patients in the $80-/ 40-\mathrm{mg}$ group. Baseline characteristics were similar except for greater rates of rectal/anal CD in the $160-/ 80-\mathrm{mg}$ group and greater use of concomitant steroids in the $80-/ 40-\mathrm{mg}$ group. Compared with the 80-/40-mg group, the 160-/80-mg group had a greater percentage of time in remission from Week $0-52(36 \%$ vs $25 \% ; \mathrm{p}<0.05, \mathrm{NRI})$, significantly fewer hospitalisations per patient $(0.09$ vs $0.23 ; \mathrm{p}<0.05)$, and significantly fewer CD-related hospitalisations ( 0.07 vs $0.18 ; \mathrm{p}<0.05$ ). Patients in the $160-/ 80-\mathrm{mg}$ group were significantly more likely to be in remission during Weeks 4 to 52 than were patients in the $80-/ 40$-mg group after adjusting for baseline characteristics (adjusted OR 4.8; $\mathrm{p}<0.001$ ). LOCF results for remission analysis were consistently similar. The 160-/80-mg regimen did not appear to lead to a higher rate of AEs.

Conclusion The 160-/80-mg induction regimen of adalimumab was associated with a greater likelihood of remission, more time in remission, and fewer hospitalisations during eow maintenance therapy compared with the $80-/ 40-\mathrm{mg}$ regimen.

Competing interests J-F Colombel Consultant for: Abbott, Speaker bureau with: Abbott, P Rutgeerts Grant/Research Support from: Abbott, Consultant for: Abbott, Speaker bureau with: Abbott, Conflict with: Abbott, W Sandborn Grant/Research Support from: Abbott, Consultant for: Abbott (fees paid to Mayo), W Reinisch Consultant for: Abbott, Conflict with: Abbott, E Loftus Jr Grant/Research Support from: Abbott, Consultant for: Abbott, J Tang Consultant for: Abbott, Employee of: Analysis Group, P Pollack Shareholder with: Abbott, Employee of: Abbott, M Yang Shareholder with: Abbott, Employee of: Abbott, S Patel Shareholder with: Abbott, Employee of: Abbott, B Huang Shareholder with: Abbott, Employee of: Abbott, J Chao Shareholder with: Abbott, Employee of: Abbott, P Mulani Shareholder with: Abbott, Employee of: Abbott.

\section{PWE-255 5-ASA ENHANCES DUOX2 EXPRESSION IN ACTIVE ULCERATIVE COLITIS: A RISK FOR COLORECTAL CANCER?}

doi:10.1136/gutjnl-2012-302514d.255

${ }^{1} \mathrm{~T}$ Macfie, ${ }^{* 1} \mathrm{~A}$ Parker, ${ }^{1} \mathrm{~A}$ Nijhuis, ${ }^{1} \mathrm{~T}$ Boitsova, ${ }^{1} \mathrm{~N}$ Suraweera, ${ }^{1} \mathrm{R}$ Jeffery, ${ }^{1} \mathrm{R}$ Poulsom, ${ }^{2} \mathrm{~J}$ Lindsay, ${ }^{1} \mathrm{~A}$ Silver. ${ }^{1}$ Centre for Digestive Diseases, Blizard Institute, Barts and The London School of Medicine and Dentistry, London, UK; ${ }^{2}$ Digestive Diseases Clinical Academic Unit, Blizard Institute, Barts and The London School of Medicine and Dentistry, London, UK

Introduction Colonic DUOX2 expression produces hydrogen peroxide, a reactive oxygen species (ROS), which is up-regulated in active ulcerative colitis (UC). Overproduction of hydrogen peroxide amplifies ROS-induced genetic damage and causes cellular transformation which may explain the increased colorectal cancer (CRC) risk associated with chronic UC. Mesalazine (5-ASA) has been shown to be chemo-preventative for UC associated CRC and scavenges ROS. Here, we aimed to identify and investigate the effect of 5-ASA on DUOX2 expression using human rectal cancer cell lines and mucosal tissue biopsies.

Methods Mucosal biopsies were taken from 35 patients with UC and 24 patients with normal colons for in vivo experiments, and 24 patients with UC and 14 patients with normal colons for ex vivo experiments. Total RNA was extracted and quantitative real-time PCR used to calculate expression of DUOX2. Cytometric bead array technology was used on ex vivo culture supernatants to measure cytokine profiles. In situ hybridisation for DUOX2 expression was performed on sections from eight matched pairs of non-inflamed/ inflamed biopsies and five matched pairs of non-inflamed/inflamed/ dysplasia biopsies from UC patients. Human rectal cancer cells were 
used for quantitative real-time PCR, with multiparameter flow cytometry utilised to measure hydrogen peroxide levels, apoptosis, DNA damage and cell proliferation.

Results DUOX2 is expressed throughout the colonic epithelium, is upregulated in active compared to quiescent ulcerative colitis and also in areas of UC associated dysplasia. In the setting of intestinal inflammation, but not in quiescent disease, 5-ASA enhances DUOX2 expression in vivo and ex vivo. As expected, 5-ASA was found to suppress cytokine (IL-6 and IL-8) production during an inflammatory flare and to maintain low cytokine levels during remission. The addition of 5-ASA in vitro led to upregulation of DUOX2 and elevated levels of hydrogen peroxide, DNA damage and apoptosis. These effects were further enhanced in a setting of hypoxia.

Conclusion We have shown that 5-ASA over stimulates DUOX2 expression in the setting of inflammation and hypoxia, but not in quiescent disease. Importantly, this suggests that during a flare 5ASA could act as a carcinogen rather than a chemo-preventative drug. Further investigations to confirm the functional relevance of DUOX2 up-regulation in the colonic mucosa of patients with active UC is indicated.

Competing interests T Macfie Grant/Research Support from: SHIRE, A Parker: None declared, A Nijhuis: None declared, T Boitsova: None declared, N Suraweera: None declared, R Jeffery: None declared, R Poulsom: None declared, J Lindsay Grant/Research Support from: SHIRE, A Silver: None declared.

\section{PWE-256 INTESTINAL INFLAMMATION REGULATES RETINOIC ACID DEPENDENT IMPRINTING OF GUT TROPISM BY DENDRITIC CELLS INDEPENDENTLY OF RALDH EXPRESSION}

doi:10.1136/gutjnl-2012-302514d.256

${ }^{1} \mathrm{~T}$ J Sanders, ${ }^{*}{ }^{1} \mathrm{~N}$ E McCarthy, ${ }^{1} \mathrm{E}$ Giles, ${ }^{2} \mathrm{~J} 0$ Lindsay, ${ }^{1} \mathrm{~A}$ J Stagg. ${ }^{1}$ Centre for Immunology and Infectious Disease, Blizard Institute, London, UK; ${ }^{2}$ Digestive Diseases Clinical Academic Unit, Barts \& The London School of Medicine \& Dentistry, London, UK

Introduction In the mouse, tissue-specific expression of retinaldehyde dehydrogenase (RALDH) enzymes by CD103+ intestinal dendritic cells (DC) enables them to generate all-trans retinoic acid (RA) and thereby imprint a gut-tropic phenotype on T cells via induction of homing receptors including $\alpha 4 \beta 7$ integrin. In health, RA from $\mathrm{CD} 103+$ also enhances their generation of Treg, contributing to intestinal homeostasis. In murine models of inflammatory bowel disease (IBD) RALDH expression by CD103+ DC is reduced but little is known about the function of RA in the human intestine. The aim of this study was to determine whether factors present in the healthy and inflamed human intestine regulate RA generation and activity.

Methods Conditioned media (CM) were generated by culture of intestinal biopsies from healthy individuals and IBD patients (inflamed/non-inflamed regions). DC were differentiated from monocytes using GM-CSF and IL-4 in the presence or absence of CM. Expression of RA-generating enzymes was assessed by qRTPCR and RALDH activity determined using the Aldefluor assay. Induction of $\alpha 4 \beta 7$ following activation of naive allogeneic CD4+ T cells was determined by flow cytometry.

Results Activation of naive CD4+ T cells by human monocytederived DC resulted in RA-dependent upregulation of $\alpha 4 \beta 7$. These DC possessed retinal-inhibitable Aldefluor activity and expressed both alcohol dehydrogenase $(R D H 10)$ and RALDH $(R A L D H 1,2,3)$ enzymes required for the generation of RA from retinol via retinal. Aldefluor activity was regulated by GM-CSF and RA, and reflected predominately the activity of RALDH2 as suggested by qRT-PCR analysis of sorted Aldefluor+ DC. CM significantly suppressed Aldefluor activity $(p<0.0001)$ irrespective of whether generated from healthy or IBD tissue (inflamed or non-inflamed). The inhib- itory effect of CM generated from healthy tissue could be partially reversed with the prostaglandin E2 (PGE2) EP-2 receptor antagonist AH6809 but this effect was less marked with CM from IBD tissue suggesting the involvement of distinct RALDH regulators. Although the effects of inflamed and non-inflamed CM on Aldefluor activity were similar, DC differentiated in the presence of inflamed $C M$ induced significantly higher $(\mathrm{p}<0.05)$ levels of CD4 T cell $\alpha 4 \beta 7$ expression.

Conclusion Factors generated in the human intestinal mucosa limit RALDH activity in DC and may thereby impact upon their generation of RA. Factors other than PGE2 are involved particularly in inflamed tissue. Intestinal mediators influence the imprinting of gut tropism independently of effects on RA-generating enzymes. Manipulation of RA availability may offer new therapeutic options in IBD.

Competing interests None declared.

\section{PWE-257 THE ROLE OF RDH10 AND RALDH ENZYMES IN RETINOIC ACID-MEDIATED IMMUNE REGULATION BY ANTIGEN PRESENTING CELLS IN THE HUMAN INTESTINE}

doi:10.1136/gutjnl-2012-302514d.257

${ }^{1} \mathrm{~T}$ J Sanders, ${ }^{*} \mathrm{~N}$ E McCarthy, ${ }^{1} \mathrm{E}$ M Giles, ${ }^{2} \mathrm{~J} 0$ Lindsay, ${ }^{1} \mathrm{~A} J$ Stagg. ${ }^{1}$ Centre for Immunology and Infectious Disease, Blizard Institute, London, UK; ${ }^{2}$ Digestive Diseases Clinical Academic Unit, Barts \& The London School of Medicine \& Dentistry, London, UK

Introduction All-trans retinoic acid (RA) has emerged as an important immunoregulatory molecule with specific functions in the intestine. RA is generated enzymatically by the sequential action of alcohol dehydrogenase $(\mathrm{ADH})$ and retinaldehyde dehydrogenase (RALDH) enzymes. In the mouse, RALDH expression is confined to $\mathrm{CD} 103+$ intestinal dendritic cells (DC) giving this subset the unique ability to mediate RA-dependent functions such as the imprinting of gut tropism on T cells. RALDH activity is downregulated in mouse models of inflammatory bowel disease (IBD) but little is known about its role in human intestine. The aim of this study was to assess RA-dependent immune regulation by human intestinal antigen presenting cells (APC).

Methods Lamina propria mononuclear cells (LPMC) were extracted from intestinal biopsies by enzymatic digestion and analysed by multicolour flow cytometry. Purified subsets of intestinal APC were obtained by immunomagnetic or FACS sorting. Expression of $A D H$ and RALDH enzymes was quantified by quantitative RT-PCR and RALDH activity assessed using the Aldefluor assay. The ability to induce the gut homing receptor $\alpha 4 \beta 7$ on T cells was assessed by flow cytometry following stimulation of CFSE-labelled naïve CD4+ T cells.

Results Induction of the gut homing receptor $\alpha 4 \beta 7$ on human T cells was RA dependent. Intestinal myeloid APC were potent stimulators of naive CD4+ T cells and induced high levels of $\alpha 4 \beta 7$. Both of these activities were attributed to the CD103+ APC fraction. However, analysis of ex vivo intestinal populations revealed RALDH activity in all myeloid APC populations studied: CD103+ and CD103- DC identified as HLA-DR+CD11c+Lin- (Lin=anti-CD3,14,16,19,34) as well as HLA-DR+CD11c+Lin+ monocyte-like cells. Lymphocytes had little or no Aldefluor activity. Comparison of intestinal DC from healthy controls and IBD patients showed similar RALDH activity in inflamed and non-inflamed tissue. RALDH activity was equivalent in $\mathrm{CD} 103+$ and $\mathrm{CD} 103-\mathrm{DC}$. In contrast, the $\mathrm{ADH}$ RDH10 was expressed at levels 4.5-fold higher in CD103+ DC.

Conclusion As in the mouse, RA-mediated induction of $\alpha 4 \beta 7$ is a property of the CD103+ subset of intestinal DC. However in divergence from murine data, this property is associated with restricted expression of the $\mathrm{ADH}$ enzyme $\mathrm{RDH} 10$ rather than 